\title{
Human RPE Melanosomes Protect from Photosensitized and Iron-Mediated Oxidation but Become Pro-oxidant in the Presence of Iron upon Photodegradation
}

\author{
Bartosz Różanowski, ${ }^{1}$ Janice M. Burke, ${ }^{2}$ Michael E. Boulton, ${ }^{3}$ Tadeusz Sarna,${ }^{4}$ and \\ Matgorzata Różanowska ${ }^{4,5}$
}

Purpose. To determine the effects of human retinal pigment epithelial (RPE) cell pigment granules on photosensitized and iron ion-mediated oxidation and the effect of the photodegradation of melanosomes on their antioxidant properties.

METHODs. RPE cells were isolated from human and bovine eyes; pigmented and nonpigmented bovine retinal pigment epithelia were isolated separately. Melanosomes, melanolipofuscin, and lipofuscin granules were isolated from human RPE donors older than 60 . Melanosomes were photodegraded by exposure to blue light. Oxidation of RPE cells or of linoleate was induced by iron/ascorbate in the presence and absence of pigment granules. The photosensitized oxidation of histidine was induced by blue light irradiation of cationic porphyrin. The progress of oxidation was monitored by electron spin resonance oximetry.

RESULTS. Iron/ascorbate induced rapid oxidation in suspensions of nonpigmented bovine RPE cells. The rates of oxidation were diminished approximately four times in suspensions of pigmented bovine RPE cells. Adding bovine melanosomes or synthetic melanin to nonpigmented bovine RPE cells resulted in a concentration-dependent decrease in the rate of oxidation to levels similar to those of pigmented bovine retinal pigment epithelium. Human melanosomes exerted a concentration-dependent inhibitory effect on photosensitized and iron-mediated oxidation. Photodegradation of human melanosomes led to loss of the inhibitory effect on iron-mediated oxidation, whereas their ability to inhibit photosensitized oxidation was enhanced.

Concuusions. Human melanosomes act as effective antioxidants by preventing iron ion-induced oxidation. Photodegradation of

From the ${ }^{1}$ Department of Genetics and Cell Biology, Institute of Biology, Pedagogical Academy of Kraków, Kraków, Poland; ${ }^{2}$ Department of Ophthalmology, Medical College of Wisconsin, Milwaukee, Wisconsin; ${ }^{3}$ Department of Ophthalmology and Visual Sciences, The University of Texas Medical Branch, Galveston, Texas; ${ }^{4}$ Department of Biophysics, Faculty of Biochemistry, Biophysics and Biotechnology, Jagiellonian University, Kraków, Poland; and ${ }^{5}$ Cardiff Vision Institute, School of Optometry and Vision Sciences, Cardiff University, Cardiff, United Kingdom.

Supported by Polish Ministry of National Education and Science Grants PB 6PO4A04423, 6PO4A06217, and 4PO5A06211; National Institutes of Health Grants R01 EY013722, and P30 EY01931; and unrestricted grants from Research to Prevent Blindness, Inc. to the Medical College of Wisconsin and Cardiff University.

Submitted for publication January 7, 2008; revised February 11, 2008; accepted May 2, 2008.

Disclosure: B. Różanowski, None; J.M. Burke, None, M.E. Boulton, None; T. Sarna, None; M. Różanowska, None

The publication costs of this article were defrayed in part by page charge payment. This article must therefore be marked "advertisement" in accordance with 18 U.S.C. $\$ 1734$ solely to indicate this fact

Corresponding author: Małgorzata Różanowska, Cardiff Vision Institute, School of Optometry and Vision Sciences, Cardiff University, Maindy Road, Cardiff CF24 4LU, UK; rozanowskamb@cf.ac.uk. melanosomes results in the loss of these antioxidant properties while it preserves their ability to deactivate cationic photosensitizers. (Invest Ophthalmol Vis Sci. 2008;49: 2838-2847) DOI:10.1167/iovs.08-1700

$\mathbf{R}$ etinal pigment epithelial (RPE) melanosomes are organelles enclosed by a lipid membrane that contain the brown-black pigment melanin synthesized on a protein matrix. ${ }^{1,2}$ Melanin exhibits several potentially antioxidant properties, such as sequestration of redox-active metal ions, scavenging of free radicals, and thermal deactivation of electronically excited states of photosensitizers and oxygen. ${ }^{1,3}$

Indeed, several studies have shown that porcine or bovine RPE melanosomes can offer substantial protection against photosensitized oxidation mediated by a cationic photosensitizer ${ }^{4}$ and iron ion-mediated generation of hydroxyl radical or lipid peroxidation. ${ }^{4-6}$ However, some of these studies also showed that photobleaching of melanosomes results in a reduced capacity to bind metal ions or to offer protection against metal ion-dependent hydrogen peroxide decomposition and lipid peroxidation. ${ }^{5,6}$ Previous studies showed that photobleaching of melanosomes mimics several changes observed with the aging of RPE melanosomes. ${ }^{5-8}$ Age-related changes in chemical properties of RPE melanosomes $^{9-11}$ suggest the possibility that their cation-binding properties also change with age.

The aims of this study were to determine the ability of RPE melanosomes to protect from iron-mediated oxidation in a cellular system, to test whether aged human RPE melanosomes retain the ability to protect from photosensitized and ironmediated oxidation, and to determine the effects of melanosome photodegradation on their antioxidant properties. To compare the iron-induced oxidation of retinal pigment epithelium in the presence and absence of RPE melanosomes and synthetic melanin, we used bovine RPE cells isolated from the pigmented part of the fundus and from the tapetum, where cells do not contain melanin. We also tested the ability of human melanosomes from 60- to 90-year-old donors to inhibit photosensitized and iron-mediated lipid peroxidation and compared them with two other human RPE pigment granules, melanolipofuscin and lipofuscin. Finally, we tested the effect of photodegradation on the ability of human melanosomes to inhibit iron-mediated lipid peroxidation and photosensitized oxidation mediated by a cationic photosensitizer.

\section{Materials AND Methods}

\section{Chemicals}

Chemicals, of reagent grade or better, were purchased from SigmaAldrich (Steinheim, Germany), or Merck (Darmstadt, Germany) and were used as supplied unless stated otherwise. The 4-protio-3-carbamoyl-2,2,5,5-tetraperdeuteromethyl-3-pyrroline-1-yloxy (mHCTPO) was a generous gift of H. J. Halpern (University of Chicago, Chicago, IL). Phosphate-buffered saline (PBS) without calcium and magnesium was treated with chelating resin (Chelex 100; Sigma-Aldrich) before 
use to minimize the content of metal ions. Synthetic dopa-melanin was prepared by autooxidation of dihydroxyphenylalanine (dopa), as previously described. ${ }^{12}$

\section{RPE Cell Isolation}

Human eyes with no reported abnormalities were obtained from the Wisconsin Lion's Eye Bank less than 24 hours after donor death (all donors were Caucasian). Bovine eyes were from a local slaughterhouse. RPE cells were isolated as described previously, and pigmented and nonpigmented cells of bovine eyes were isolated separately. ${ }^{10}$ Protein concentration was determined by the Lowry method. ${ }^{13}$

\section{Isolation of Melanosomes, Melanolipofuscin, and Lipofuscin Granules}

Pigment granules were isolated from human RPE cells from 60- to 90-year-old donors or from pigmented bovine retinal pigment epithelium and were purified as previously described. ${ }^{14}$ Given that $1 \mathrm{mM}$ EDTA was used for retinal pigment epithelium homogenization and 0.1 mM EDTA was used for further centrifugation steps, most of the iron ions bound endogenously to the melanosomes were removed during the isolation procedure. Finally, purified melanosomes were washed in PBS without EDTA. The number of the human pigment granules was determined by counting in a hemocytometer.

\section{Determination of Free Radical Content in Melanin}

The number of free radicals in melanin was determined by an electron spin resonance (ESR) assay ${ }^{8,11}$ in which the ESR spectra of melanin samples acidified to $\mathrm{pH} 1.0$ were measured at liquid nitrogen temperature, in the g-value of approximately 2.00 region (ESP 300E ESR spectrometer; Bruker Instruments. Billerica, MA) operating at X-band and equipped with $100-\mathrm{kHz}$ field modulation. Apparatus settings were as follows: microwave power $0.02 \mathrm{~mW}$, modulation amplitude $2.0 \mathrm{G}$, and scan range $50 \mathrm{G}$. ESR signal intensities of RPE cells and isolated pigment granules were compared under identical experimental conditions with the ESR signal of dopa-melanin.

\section{Photo-Induced Degradation of Melanosomes}

Isolated melanosomes in suspension in PBS were photodegraded at $10^{\circ} \mathrm{C}$ in a thermostated optical chamber with constant stirring, using a magnetic stirrer. Samples were irradiated up to 70 hours with a compact arc high-pressure mercury lamp (Photomax $200 \mathrm{~W}$; Oriel Co., Stratford, CT) with light filtered by a combination of cutoff and broadband filters transmitting blue light 390 to $490 \mathrm{~nm}\left(22 \mathrm{~mW} / \mathrm{cm}^{2}\right)$. Light intensity was measured by determining fluence rates with a radiometer (model 65A; Yellow Springs Instrument Co., Yellow Springs, OH).

\section{Solubilization of Methyl Linoleate in Micelles}

Micelles were prepared fresh before experiments. Methyl linoleate was added (final concentration, $75 \mathrm{mM}$ ) to an argon-saturated solution of $10 \mathrm{mM}$ Triton X-100 in PBS, vortexed, and kept on ice under argon until use.

\section{Induction of Oxidation by $\mathrm{Fe} /$ Ascorbate}

Fe(III) was chelated with adenosine diphosphate (ADP) by solubilization of ferric chloride in the presence of $5 \mathrm{~mol}$ eq excess of ADP at $\mathrm{pH}$ 1.0 (to prevent iron precipitation), followed by a gradual increase in $\mathrm{pH}$ by the addition of $\mathrm{NaOH}$ to $\mathrm{pH} 7.2$, producing a stock solution of $0.5 \mathrm{mM}$ Fe-ADP. Several incubation times of the Fe-ADP complex with melanosomes were tested. No difference in inhibitory action was detectable between 5 minutes and longer incubation times up to 60 minutes, Therefore, in all further experiments, melanosomes were incubated for 5 minutes with Fe-ADP before the addition of $0.2 \mathrm{mM}$ ascorbate and RPE homogenates or linoleate and the measurement of oxygen uptake.

\section{Monitoring of Oxidation by Electron Spin Resonance Oximetry}

The kinetics of oxygen concentration changes were measured by ESR oximetry $^{10,14,15}$ using $0.1 \mathrm{mM}$ mHCTPO as the nitroxide spin probe. Initial rates of oxygen uptake were obtained by determining the slope of initial oxygen uptake, where the oxygen concentration decreased linearly with time.

\section{Oxidation Induced by a Cationic Photosensitizer}

Histidine was used, a substrate easily susceptible to oxidation by singlet oxygen, an electronically excited state of molecular oxygen that can be generated by photoexcitation of photosensitizers such as tetra(4-N,N,N,N-trimethylanilinium)porphyrin (TAP) under aerobic conditions. ${ }^{16,17}$ To induce photosensitized oxidation of histidine, a solution of $0.088 \mathrm{mM}$ TAP in the presence of $2 \mathrm{mM}$ histidine was irradiated with blue light in a flat quartz cell $(0.25 \mathrm{~mm}$ optical path length) directly in a resonant cavity of the ESR spectrometer to allow concomitant monitoring of oxygen concentration by ESR oximetry. ${ }^{4}$ Irradiance levels inside the ESR resonant cavity were monitored by a calibrated silicon photodiode (Hamamatsu Photonics K.K., Shizuoka, Japan).

\section{Statistical Analysis}

All experiments were performed at least in triplicate. Unless stated otherwise, experimental points and error bars in figures represent mean and standard deviation (SD), respectively. Statistically significance differences between means were determined using the Student's $t$-test, with differences of $P<0.05$ considered significant. First-order polynomial regressions were performed using appropriate software (SigmaPlot9.01; Systat Software Inc., London, UK).

\section{Results}

\section{Melanin as the Main Inhibitor of Iron-Mediated Oxidation in the Retinal Pigment Epithelium}

Iron (III) ions chelated with ADP are easily reduced by ascorbate, and the mixture of Fe(II)-ADP and Fe(III)-ADP is expected to interact with hydroperoxides, leading to their decomposition and resulting in the formation of lipid-derived radicals or hydroxyl radicals, each of which can initiate a free radical chain of lipid peroxidation. ${ }^{18}$ Indeed, as expected, the addition of Fe-ADP/ascorbate to a suspension of nonpigmented bovine RPE cells induced rapid oxidation, leading to the depletion of about half the solubilized oxygen within approximately 12 minutes (Fig. 1A). In the absence of exogenous ascorbate, the rate of oxygen uptake was 13.7 times slower than in its presence, whereas in the absence of exogenous Fe-ADP, the rate of oxidation was negligible (Figs. 1A, 1B).

The initial rate of oxidation was approximately 4.1 times smaller in suspensions of pigmented bovine RPE cells than in nonpigmented cells of equivalent protein concentration (Fig. 1). The addition of increasing concentrations of bovine melanosomes to the suspension of nonpigmented bovine RPE cells resulted in a concentration-dependent decrease in the rate of oxygen uptake (Fig. 1). In the presence of bovine melanosomes at a concentration corresponding to melanin concentration in pigmented RPE cells $(0.03 \mathrm{mg}$ melanin $/ \mathrm{mL})$, the kinetics of oxygen uptake in homogenates of nonpigmented RPE cells were similar to those in homogenates of pigmented RPE cells. Oxidation was inhibited even further when the concentration of bovine melanosomes was doubled to $0.06 \mathrm{mg}$ melanin $/ \mathrm{mL}$. Interestingly, $0.06 \mathrm{mg} / \mathrm{mL}$ synthetic dopa-melanin exerted an inhibitory effect on oxygen uptake similar to that of bovine melanosomes with the same concentration of melanin. 

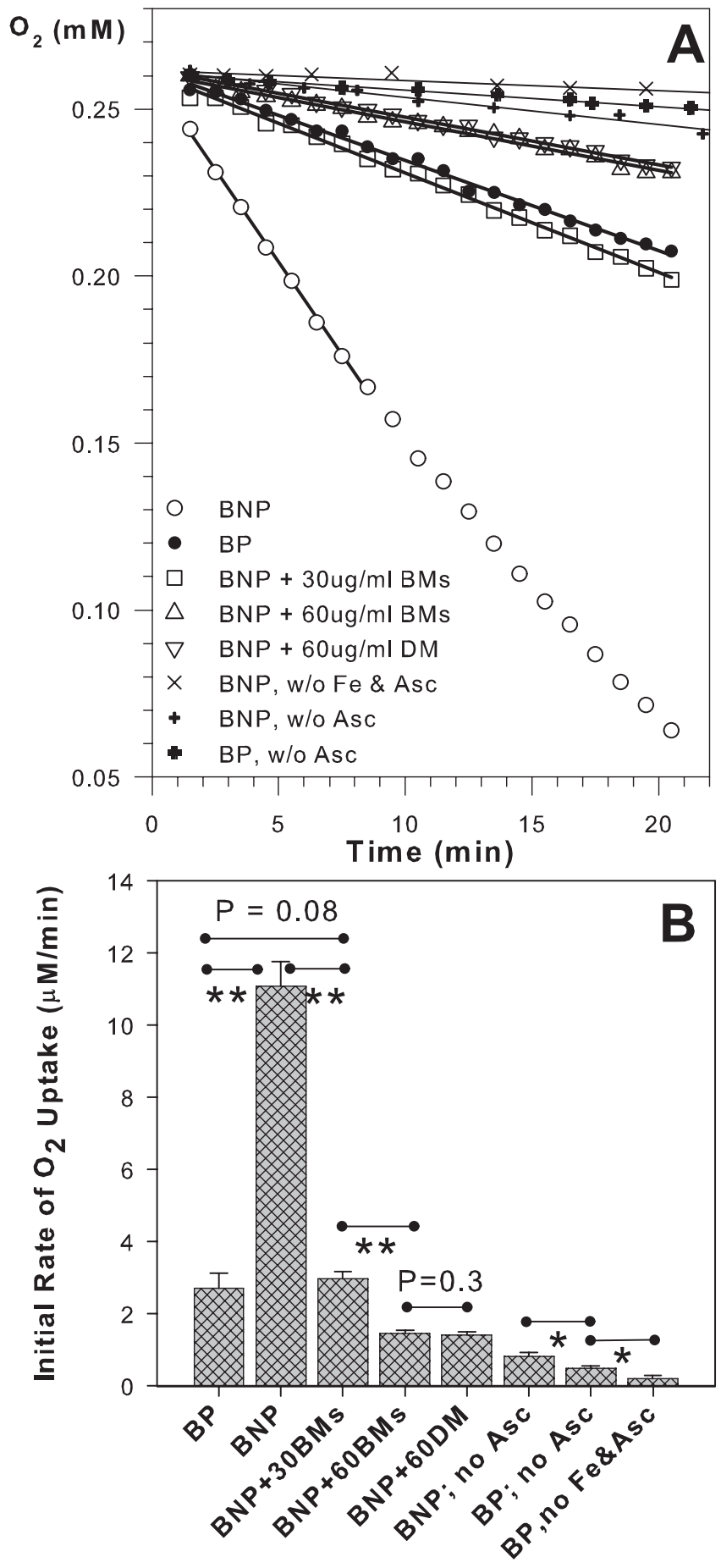

FIGURE 1. Representative kinetics (A) and mean ( \pm SD) initial rates (B) of oxygen uptake induced by Fe-ADP/ascorbate $(0.05 \mathrm{mM} / 0.20$ $\mathrm{mM}$ ) in a suspension of nonpigmented bovine RPE cells (BNP), pigmented bovine RPE cells (BP), or nonpigmented bovine RPE cells in the presence of bovine melanosomes (BMs) or synthetic dopa-melanin (DM) with indicated melanin concentrations. ${ }^{*} P<0.005$; ${ }^{* *} P<5 \times$ $10^{-6}$.

\section{Ability of Human Pigment Granules from the Old Retinal Pigment Epithelium to Inhibit Fe-Mediated Oxidation}

Because human melanosomes undergo several age-related changes, ${ }^{9-11}$ it is possible that their interaction with iron ions also changes, reducing the ability of the melanosome to inhibit iron ion-induced lipid peroxidation. Human melanosomes from aged donors exerted a dose-dependent inhibitory effect on Fe-ADP/ascorbate-induced oxygen uptake in the presence of linoleate (Fig. 2). The inhibition became greater at increasing concentrations of human melanosomes but only up to 0.6 billion granules $/ \mathrm{mL}$, corresponding to $0.03 \mathrm{mg} / \mathrm{mL}$ melanin. Above 0.6 billion granules/mL, no further inhibition was detected. In the absence of linoleate, samples with human melanosomes exhibited substantial oxygen uptake, indicating that human melanosomes were themselves a susceptible substrate for oxidation (Fig. 2).

In comparison with bovine melanosomes, human melanosomes proved significantly less effective than bovine melanosomes at inhibiting Fe-mediated oxidation of linoleate at concentrations corresponding to the same melanin concentration (Fig. 3). Bovine melanosomes inhibited the rate of Fe-ADP/ ascorbate-induced oxygen uptake 10.5-fold in suspensions of linoleate in micelles, whereas human melanosomes inhibited the rate approximately sixfold (Figs. 3B, 3C).

Melanin in the adult human retinal pigment epithelium is present in melanosomes and in the complex pigment granules melanolysosomes and melanolipofuscin. ${ }^{19-22}$ Melanolipofuscin, tested at the same concentration of granules as human melanosomes, exhibited some inhibitory effect by inhibiting the oxidation of linoleate by approximately 34\% (Figs. 3B, 3D). Lipofuscin did not exert a significant effect on Fe-ADP/ascorbate-induced oxygen uptake in the presence of linoleate (Figs. 3B, 3D).

Because of their protein and lipid content, pigment granules from human retinal pigment epithelium may themselves be susceptible to iron-induced lipid peroxidation. Indeed, incubation of Fe-ADP/ascorbate in the absence of linoleate but in the presence of bovine or human melanosomes led to a 1.4-fold and a 2.7 -fold increase, respectively, in the initial rate of oxygen uptake in comparison with samples without pigment granules (Figs. 3A, 3C). Almost identical rates of oxygen uptake were observed with the addition of Fe-ADP/ascorbate to a suspension of lipofuscin granules (Figs. 3A, 3C). Interestingly, iron-induced oxygen uptake in a suspension of melanolipofuscin granules was approximately 2.1 and 2.8 times greater than for lipofuscin and melanosomes, respectively (Figs. 3A, 3C).

\section{Effect of Photodegradation of Melanosomes on Their Inhibitory Effect on Fe-Mediated Oxidation}

It has been suggested that photooxidation may be responsible for age-related degradation of melanin and changes in its physicochemical properties. ${ }^{8}$ Photodegradation of human melanosomes with blue light for 70 hours led to a decrease in the intrinsic free radical signal of melanin by a factor of 2.3 (Fig. 4). In the absence of Fe-ADP/ascorbate, neither native nor photodegraded melanosomes exhibited a detectable oxygen uptake. On the addition of Fe-ADP/ascorbate to a suspension of melanosomes photodegraded for 12 hours, the rate of oxygen uptake was similar to that of nondegraded melanosomes (Figs. 5A, 5C). Melanosomes photodegraded for 70 hours exhibited significantly faster oxygen uptake in the presence of Fe-ADP/ ascorbate, with rates approximately 1.9 and 1.8 times greater, respectively, than nondegraded melanosomes and melanosomes degraded for 12 hours (Figs. 5A, 5C).

In the presence of linoleate, native melanosomes inhibited Fe-ADP/ascorbate-mediated oxidation by a factor of 2.4 (Figs. 5B, 5D). Not only did melanosomes degraded for 12 hours not exert a protective effect, they exhibited a small but significant pro-oxidant effect, leading to a $13 \%$ increase in the rate of oxygen uptake (Figs. 5B, 5D). Melanosomes degraded for 70 hours induced a substantial pro-oxidant effect on Fe-ADP/ ascorbate-induced oxygen uptake, increasing the rate of oxy- 

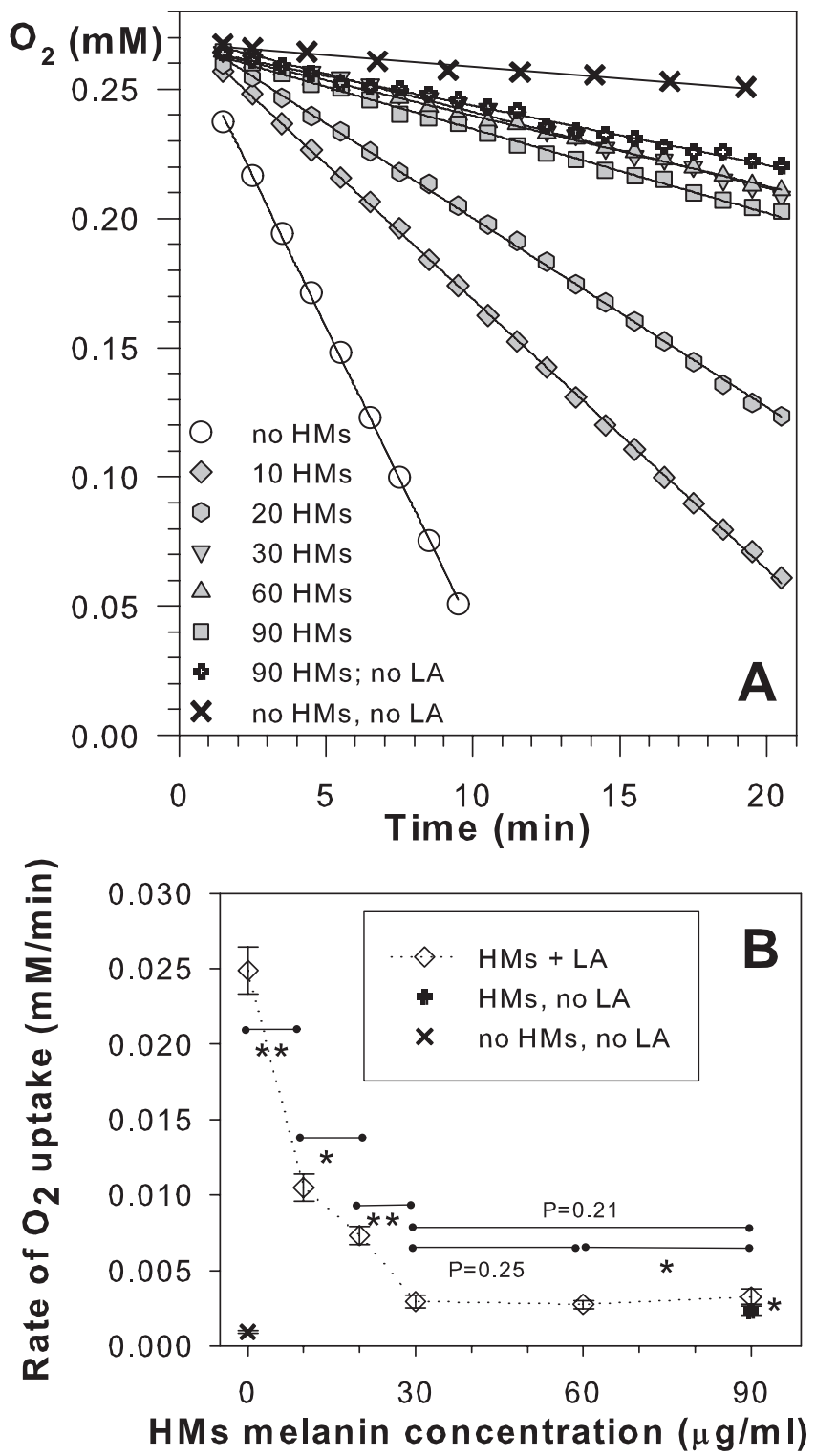

FIGURE 2. Inhibition of Fe ion-catalyzed lipid oxidation as a function of the concentration of human melanosomes (HMs). (A) Representative kinetics of oxygen depletion in a suspension of $10 \mathrm{mM}$ linoleate (LA), $0.2 \mathrm{mM}$ ascorbate, and $0.05 \mathrm{mM}$ Fe-ADP, in the presence and absence of human melanosomes at the indicated melanin concentrations. (B) Mean $( \pm \mathrm{SD})$ initial rates of oxygen uptake as a function of melanin concentration in human RPE melanosomes. ${ }^{*} P<0.05$. ${ }^{* *} P<$ $5 \times 10^{-5}$.

gen uptake by approximately $89 \%$ in comparison with samples without melanosomes and 4.6 times faster than for native melanosomes (Figs. 5B, 5D).

\section{Effect of Human Melanosomes on Photosensitized Oxidation Induced by Cationic Photosensitizer}

Next we tested the ability of aged human melanosomes to inhibit photosensitized oxidation mediated by TAP, a watersoluble cationic derivative of porphyrin known to bind to melanin, which inhibits its photosensitizing action by quenching electronically excited states. ${ }^{3,16,23-26}$ In the absence of melanin, the photoexcitation of TAP with blue light led to a photosensitized energy transfer from an excited triplet state of
TAP to oxygen, forming an excited state of oxygen known as singlet oxygen. Singlet oxygen, in turn oxidizes histidine, resulting in rapid oxygen depletion (Fig. 6). Adding human melanosomes from aged donors showed a dose-dependent inhibitory effect on photooxidation (Figs. 6A, 6C). Interestingly, photodegraded melanosomes proved more efficient at inhibiting TAP-mediated photooxidation (Figs. 6B, 6C). The 50\% inhibition was provided by degraded melanosomes at a concentration of $0.14 \times 10^{9}$ granules $/ \mathrm{mL}$, whereas a fivefold higher concentration of native melanosomes was required to provide a similar inhibitory effect (Fig. 6C).

\section{Discussion}

\section{Melanosomes Protect against Iron-Mediated Oxidation}

In several model systems that have been studied, the binding of iron to melanin provided either antioxidant protection against metal ion-mediated oxidation ${ }^{4,27,28}$ or mediated pro-oxidant effects. $^{5,6,29}$ Oxidation of melanin-bound reduced metal ions, such as $\mathrm{Fe}(\mathrm{II})$ and $\mathrm{Cu}(\mathrm{I})$, by hydrogen peroxide or oxygen leads to the formation of hydroxyl radical and superoxide, respectively. However, these products interact rapidly with the melanin itself ${ }^{30}$; therefore, the risk of free radicals escaping the melanin is very low. Moreover, oxidized metal ions such as $\mathrm{Fe}$ (III) and $\mathrm{Cu}$ (II) bound to melanin are substantially less susceptible to reduction by typical physiological electron donors such as ascorbate, NADH, and NADPH. ${ }^{1,3}$ If the Fenton-type reaction does occur, the hydroxyl radicals that are formed are scavenged in situ by melanin itself. ${ }^{1,30}$ Therefore, the binding of metal ions by melanin largely disables their redox cycling, and melanin-bound metal ions may be substantially less damaging to cellular components. ${ }^{1,3,29,31-33}$

However, melanin itself can reduce Fe(III) or $\mathrm{Cu}(\mathrm{II}) \mathrm{com}$ plexed by a strong chelator such as $\mathrm{DTPA}^{34}$ or, under more biologically relevant conditions, by a weak chelator such as citrate in the excess of metal ions compared with melaninbinding sites. ${ }^{28}$ Photoexcitation of melanin in the presence of oxygen leads to the generation of superoxide radical anions, ${ }^{3}$ which in turn can reduce iron or copper ions. ${ }^{18}$ Thus, the entire process, mediated by melanin, can be complex, with the net antioxidant or pro-oxidant result depending on many factors, such as relative concentration of metal ions, small molecular weight chelators and melanin-binding sites, presence of oxygen, and irradiation conditions. Under special conditions, such as the presence of EDTA, redox cycling of metal ions by melanin may lead to pro-oxidant effects such as those demonstrated for sepia melanosomes enriched with iron or copper that mediate damage to extragranular DNA. ${ }^{29}$ It has also been demonstrated that low-molecular-weight melanin components leak out of iron-saturated sepia melanosomes, indicating that the aggregation state of native melanin polymer is lost. ${ }^{35}$ Lowmolecular-weight melanin components exhibit chemical properties different from those of the intact melanin polymer, and are, for example, more photoreactive. ${ }^{36}$

Some previous studies indicate that isolated RPE melanosomes can effectively protect exogenously added substrates, lipids, or salicylates from iron ion-mediated oxidation or hydroxylation. ${ }^{4-6}$ Our present results clearly demonstrate that RPE melanosomes effectively inhibit iron-mediated oxidation of RPE homogenates. Bovine pigmented and nonpigmented RPE cells provided an excellent system to prove the protective effects of melanosomes on iron-mediated oxidation in RPE homogenates. Tapetal and nontapetal retinal pigment epithelia are likely to differ in aspects other than pigmentation because of known topographic variations in the RPE monolayer. Importantly, however, on the addition of bovine melanosomes to the 

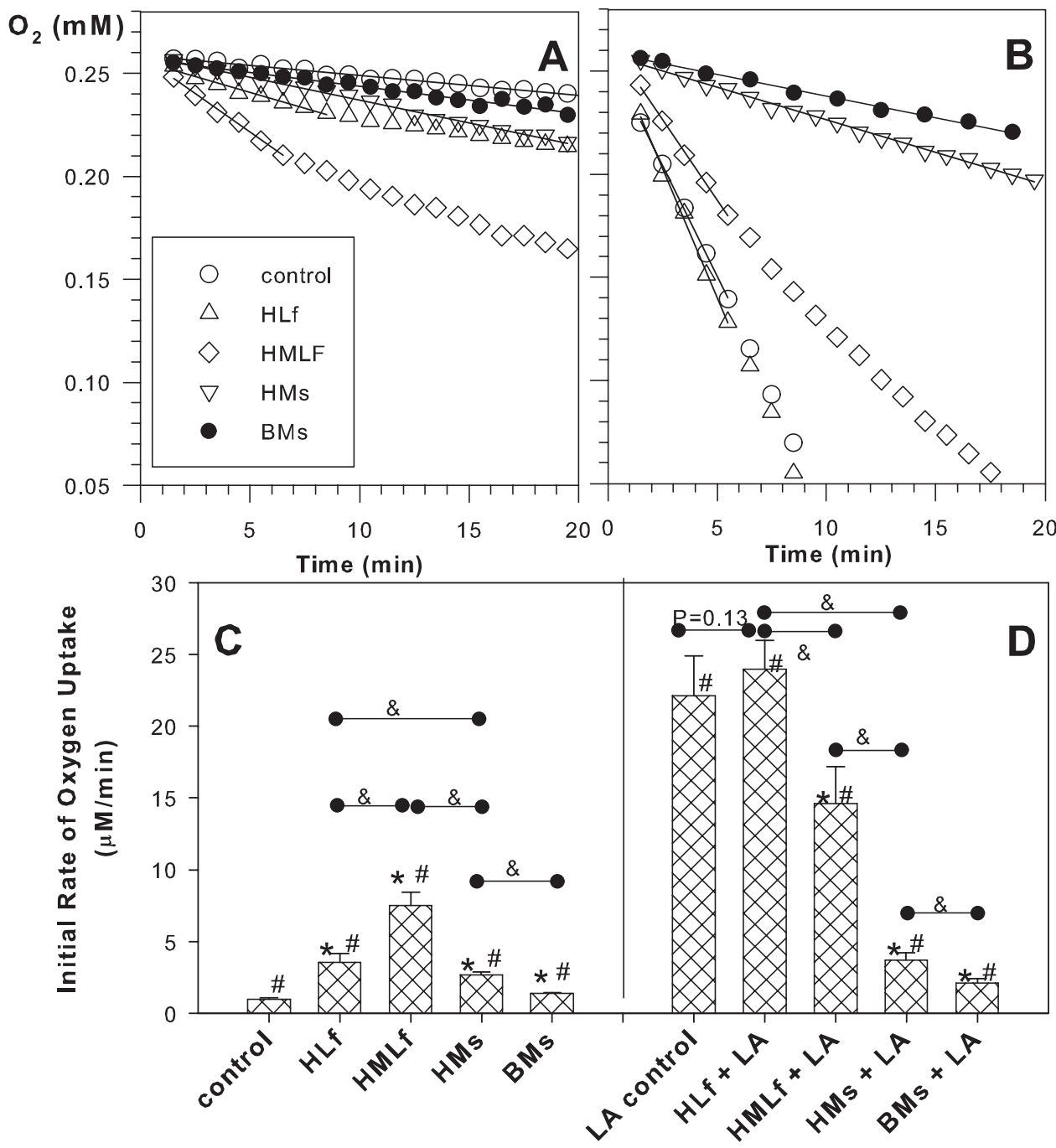

Figure 3. Comparison of the effects of human RPE pigment granules $\left(0.52 \times 10^{9}\right.$ granules $/ \mathrm{mL}$; melanosomes, HMs; melanolipofuscin, HMLF; lipofuscin, HLf) or bovine RPE melanosomes (BMs; $26 \mu \mathrm{g}$ melanin/ $\mathrm{mL}$ ) on oxygen uptake induced by Fe-ADP/ascorbate $(0.05 \mathrm{mM} / 0.2 \mathrm{mM})$ in the absence and the presence of $10 \mathrm{mM}$ linoleate (LA). (A, B) Representative kinetics of oxygen uptake in the absence (A) and the presence (B) of linoleate and mean $\pm \mathrm{SD}$ of initial rates of oxygen uptake $(\mathbf{C}, \mathbf{D})$. ${ }^{*} P<0.05$ for $t$-test comparisons of samples containing pigment granules with corresponding control samples. $\# P<0.05$ for $t$-test comparisons of corresponding samples with and without LA. $\& P<0.05$ for $t$-test comparisons of samples (๑). nonpigmented bovine RPE cells, we could diminish the rate of oxidation to that observed in pigmented bovine RPE cells. Moreover, bovine melanosomes exert protection similar to that of synthetic melanin, suggesting that melanin is the main factor responsible for the binding of $\mathrm{Fe}$ ions in the retinal pigment epithelium.

Human melanosomes have been shown to undergo agerelated changes in their photophysical properties (absorption and fluorescence), ${ }^{37}$ melanin content, ${ }^{11}$ and light-induced chemical reactivity. ${ }^{10}$ An age-related increase in the lipid component surrounding the granule ${ }^{20}$ may hinder the passage of charged ions inside. Thus, melanosome interactions with iron ions may also change with age such that melanosomes from aged donors are less competent to inhibit iron ion-induced lipid peroxidation. Yet, our investigation of the inhibitory effect of human melanosomes on Fe-ADP/ascorbate-induced oxygen uptake in the presence of linoleate demonstrated that human melanosomes from donors older than 60 offer substantial dose-dependent protection against Fe-mediated oxidation. These results indicate that, at least under our experimental conditions, age-related increases in lipid content and modifications of melanin do not substantially affect melanosomal ability to inhibit iron ion-mediated oxidation.

Thus, our results support the postulate that melanosomes may play an important antioxidant role in the retinal pigment epithelium, ${ }^{1}$ a site of continual trafficking of iron between the choroidal blood supply and photoreceptor outer seg- ments. ${ }^{38-41}$ Moreover, daily phagocytosis of photoreceptor outer segments rich in iron imposes an additional risk of iron ions escaping from binding proteins in the retinal pigment epithelium. Melanin has a large capacity for binding metal ions, and it has been documented that choroidal and RPE melanosomes do indeed accumulate substantial amounts of zinc, copper, and iron. ${ }^{4,42,43}$ Thus, RPE melanosomes may perform an antioxidant function by sequestering redox active metal ions and disabling their redox cycling or by rapid scavenging of free radicals, assuming these are generated by Fenton-type processes occurring within the melanosome. ${ }^{1}$

\section{Pro-oxidant Effects of Melanolipofuscin in Fe-Induced Oxidation}

Melanosomes are the most prominent pigment granules in the young retinal pigment epithelium, but with aging there is a progressive accumulation of lipofuscin and complex granules containing melanin and lipofuscin (melanolipofuscin). ${ }^{4,19-22}$ Interestingly, melanolipofuscin in the presence of iron ions mediates rapid oxygen uptake in the absence of additional oxidation substrate, which is approximately 2.1 and 2.8 times greater than in the presence of lipofuscin and melanosomes, respectively. Perhaps the proximity of melanin to lipofuscin, when both are enclosed within the same melanolipofuscin granule, results in the greatest susceptibility to iron-induced oxidation in comparison with lipofuscin and melanosomes. 


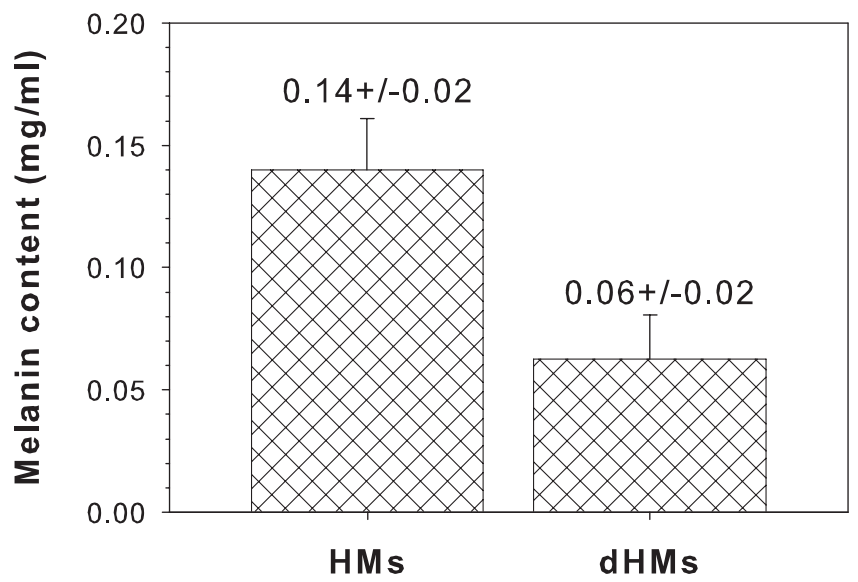

FiguRE 4. Melanin content in human melanosomes $\left(2.8 \times 10^{9}\right.$ granules/mL) before (HMs) and after (dHMs) photodegradation for 70 hours.

Perhaps the melanin in melanolipofuscin is still able to bind iron but enables its redox cycling by ascorbate. Then, because of high local availability of unsaturated lipids, oxidation may proceed more quickly than it does for melanosomes (which have fewer substrates available for oxidation and less degraded melanin) or for lipofuscin (which apparently exhibits no affinity for iron).

\section{Loss of Antioxidant Properties and Pro-oxidant Action of Melanosomes in the Presence of Iron Ions Caused by Photodegradation}

With age there is a marked decrease in RPE melanin, as demonstrated by a morphometric quantification of cell volume occupied by melanosomes, ${ }^{7,20,22}$ a decrease in the characteristic subunits of melanin, ${ }^{44,45}$ and a decrease in the content of the intrinsic free radical of melanin in RPE cells ${ }^{8}$ and in isolated RPE melanosomes. ${ }^{11}$ It has been suggested that age-related loss of RPE melanin and changes in its physicochemical properties may result from photooxidation. ${ }^{5,6,8}$ Melanin functions as a broadband filter whose absorption coefficient monotonically increases with decreasing wavelength across the infrared, visible, and ultraviolet parts of the electromagnetic spectrum. The energy of the absorbed radiation is largely converted to heat, whereas only a very small percentage of absorbed photons results in the formation of free radical, the melanin radical that on interaction with oxygen forms the superoxide radical. However, different functional groups of melanin can efficiently scavenge superoxide and reduce it to hydrogen peroxide or oxidize it back to oxygen. ${ }^{3}$ Thus, the irradiation of melanin with visible light, particularly in the presence of redox active metal ions, leads to the generation of free radicals targeting melanin itself. ${ }^{46}$

Indeed, we and others ${ }^{10,47}$ have previously shown that the photodegradation of RPE melanosomes is accompanied by the photogeneration of superoxide radical, hydrogen peroxide, and oxidation of intragranular components resulting in melanosomes with increased fluorescence, decreased concentrations of intrinsic free radicals, and increased yields of photogeneration of superoxide and photooxidation of extragranular proteins. ${ }^{5,7,8,48}$ Because these changes suggest extensive chemical changes of the melanin subunits or modifications in physicochemical interaction between components, it has been tempting to test whether photodegradation affects metal ionbinding properties and, therefore, the inhibitory effects on metal ion-mediated oxidation.
In our previous studies, the photodegradation of synthetic melanin, porcine melanosomes, and bovine melanosomes was induced by a broad spectrum of visible light ${ }^{5}$ or ultraviolet and visible light ${ }^{6}$ and resulted in a reduced capacity to bind iron ions and a loss of inhibitory effect on Fe-mediated oxidation. The wavelength dependence of photo-induced oxygen uptake indicates that human melanosomes become more susceptible to photooxidation with decreasing irradiation wavelength. ${ }^{14}$ However, because of the filtering effect of the adult human lens, almost no ultraviolet light reaches the adult retina; therefore, only light above $390 \mathrm{~nm}$ is physiologically relevant for the photodegradation of adult human melanosomes. ${ }^{49}$ Here we have demonstrated that the photodegradation of melanosomes with acute blue light decreases their ability to protect from iron-mediated oxidation and that the degraded melanosomes themselves become a susceptible target for iron-mediated oxidation. It appears that iron ions bound to the surface of partially degraded melanin are more susceptible to reduction by ascorbate, and their redox cycling will elicit oxidation. Such a scenario, in which iron binds to degraded melanin but can be easily reduced by ascorbate and the subsequently formed free radicals are scavenged within melanosomes contributing to oxygen depletion, explains the observed effects by which photodegraded melanosomes appear to be more susceptible than native melanosomes to metal ion-catalyzed oxidation mediated by ascorbate.

In our experimental setup, melanosomes were exposed to a total dose of $92.4 \mathrm{~J} / \mathrm{cm}^{2}$ blue light. Fluence rates reaching the retina exposed to daylight vary from approximately 10 to 100 $\mu \mathrm{W} / \mathrm{cm}^{2} .{ }^{50}$ Assuming that only $1 \mu \mathrm{W} / \mathrm{cm}^{2}$ blue light reaches the retina of a person older than 60 because of the screening effect of the crystalline lens, it can be roughly estimated that the human retina exposed to physiologically relevant fluence rates will accumulate such a dose over 1069 days of continual exposure to blue light. Assuming further that light exposure takes place for approximately 16 hours/day, 4.4 years would be required to achieve similar degradation of melanosomes, unless endogenous antioxidants effectively counteract the photodamage.

Another point worth consideration is metal content in aged melanosomes under physiological conditions. ${ }^{43}$ Our isolation procedure of pigment granules in the presence of EDTA removes most metal ions before photodegradation. Given that melaninbound iron or copper accelerates melanin photodegradation, ${ }^{46}$ it is likely that the dose of light required to induce substantial photodegradation in vivo is smaller than that used in our study.

It must be stressed that the melanosomes used for our study were from aged persons and therefore might already have been partially photodegraded because of lifelong exposure to light. Our previous experiments on synthetic melanin and bovine RPE melanosomes showed that after an initial period of relatively small changes in optical properties and in the intrinsic free-radical concentration of synthetic or bovine melanosomes, the next phases of bleaching and loss of intrinsic free radicals of melanin are more rapid., ${ }^{5,6,8}$ If this is the case for human melanosomes, it may be speculated that age increases the risk for photodegradative changes in melanosomes. Moreover, with age, melanosomes increase their photoreactivity; on irradiation with visible light, the photogeneration of superoxide and the overall rate of photo-induced oxidation increases. ${ }^{10}$ These data also suggest an increased susceptibility of aged melanosomes to photodegradation.

\section{Mechanistic Insight into Degradation-Dependent Changes in Melanosome Interaction with Iron Ions}

There are several possible explanations for the observed effects of photodegradation on the interaction of melanosomes with iron, by which changes in melanosome structure as a 

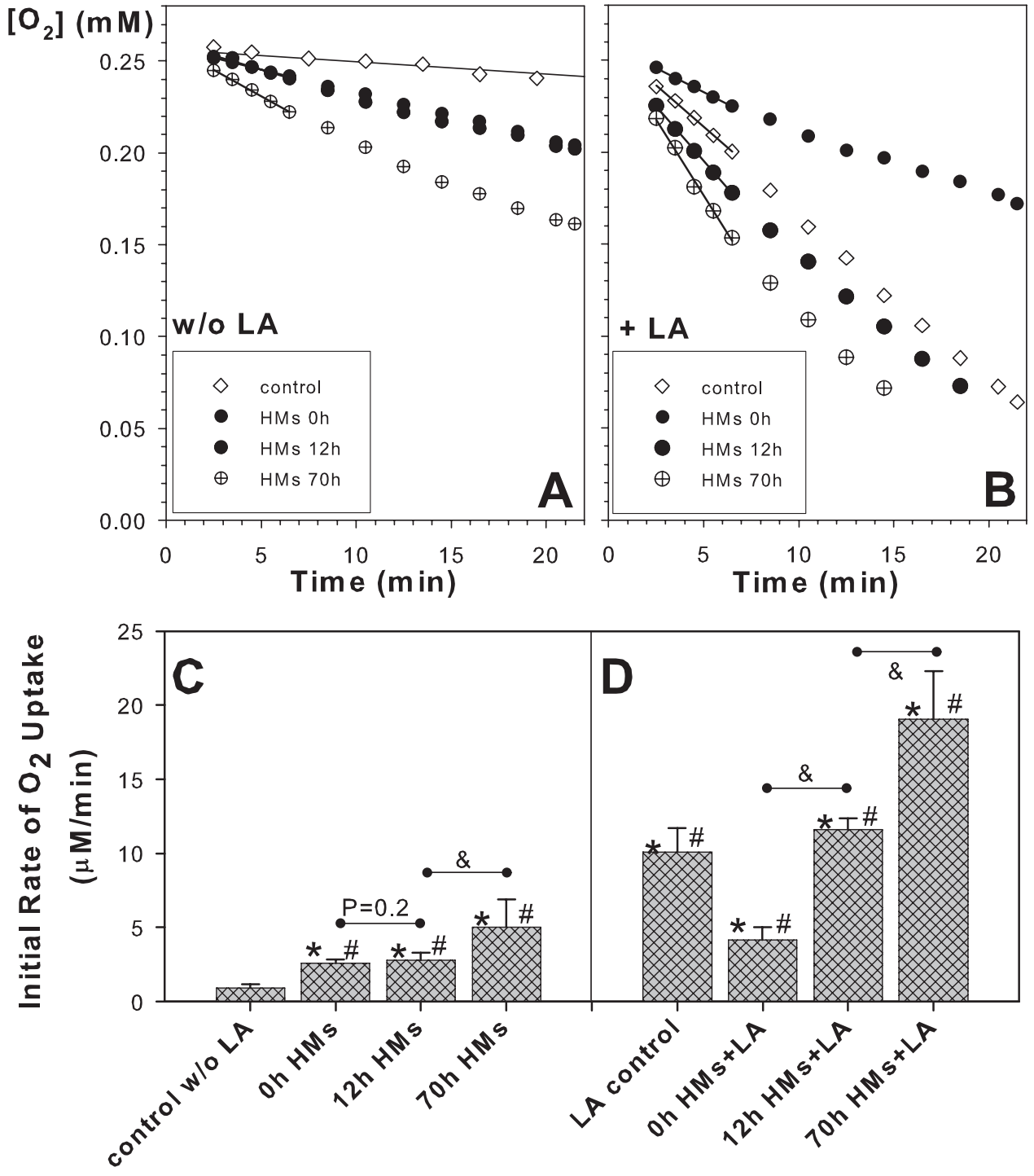

FiguRE 5. Effect of photodegradation of human melanosomes on oxygen uptake induced by Fe-ADP/ascorbate $(0.05 \mathrm{mM} / 0.2 \mathrm{mM})$ in the absence $(\mathbf{A}, \mathbf{C})$ and the presence $(\mathbf{B}$, D) of $10 \mathrm{mM}$ linoleate (LA). (A, B) Representative kinetics of oxygen depletion in control samples and samples containing human melanosomes (HMs) photodegraded for the indicated times. (C, D) Mean $( \pm S D)$ initial rates of oxygen uptake. ${ }^{*} P<$ 0.05 for $t$-test comparisons of samples containing pigment granules with corresponding control samples. $\# P<0.05$ for $t$-test comparisons of corresponding samples with and without LA. $\& P<0.05$ for $t$-test comparisons of samples $(\bullet)$. granule and melanin structure (and thus its chemical properties) must be considered. Based on published data and results obtained in this study, it can be suggested that the photodegradation of melanosomes leads to increased accessibility of melanin-binding sites for iron ions and to decreased stability of the iron complexes with melanin-binding sites modified by oxidation. This is supported by several findings.

Structural studies of changes in synthetic melanin suggest that on degradation induced by hydrogen peroxide, melanin polymer deaggregates because of the oxidative disruption of hydrogen and covalent bonds and the oxidative modification of the melanin subunits. ${ }^{51}$ As a result, there is an increased number of negatively charged carboxyl groups and a reduced number of other functional groups such as ortbo-phenols. ${ }^{51}$ These carboxyl groups may provide increased concentration of binding sites for certain cationic molecules. On the other hand, a study by Zareba et al. ${ }^{5}$ showed that photodegradation of the melanosome melanin polymer results in a decreased capacity for the binding of iron ions. These two apparently contradictory findings can be explained if we consider not only the total number of potential binding sites in melanin but also their type, which determines the stability of the formed complexes with metal ions and organic cations and the accessibility of iron ions to different melanin functional groups.
Photobleaching of melanin may increase the uptake of organic cations that are weakly bound to melanin carboxyl groups and may decrease the number of strong complexes formed between melanin bidentate ortho-phenolic sites and ferric ions. Interestingly, preferential binding of Fe(III) through coordination to o-dihydroxyl groups in Sepia melanosomes was concluded based on the results of recent Raman studies. ${ }^{32}$

An elegant study by Simon and colleagues ${ }^{32}$ demonstrated that the binding of iron ions to sepia melanosomes is not limited to the surface of the granule, and they postulated that channels must exist within the melanosome that enable trafficking of the metal ions inside the granule. We have previously shown that blue light irradiation of RPE melanosomes from older human donors leads to photooxidation of melanosomal constituents, including lipids, and that only approximately $28 \%$ of oxygen consumed accumulates as hydrogen peroxide. ${ }^{10,14}$ Transmission and scanning electron microscopy and atomic force microscopy show that photodegraded porcine melanosomes lose electron density, undergo fragmentation, and lose smoothness from their surfaces. ${ }^{5}$ Thus, it may be suggested that modification of the melanosome surface and changes in the number and type of melanin-binding sites, because of photo-induced oxidation, may facilitate the penetration of charged molecules, such as iron ions or ascorbate, toward the 

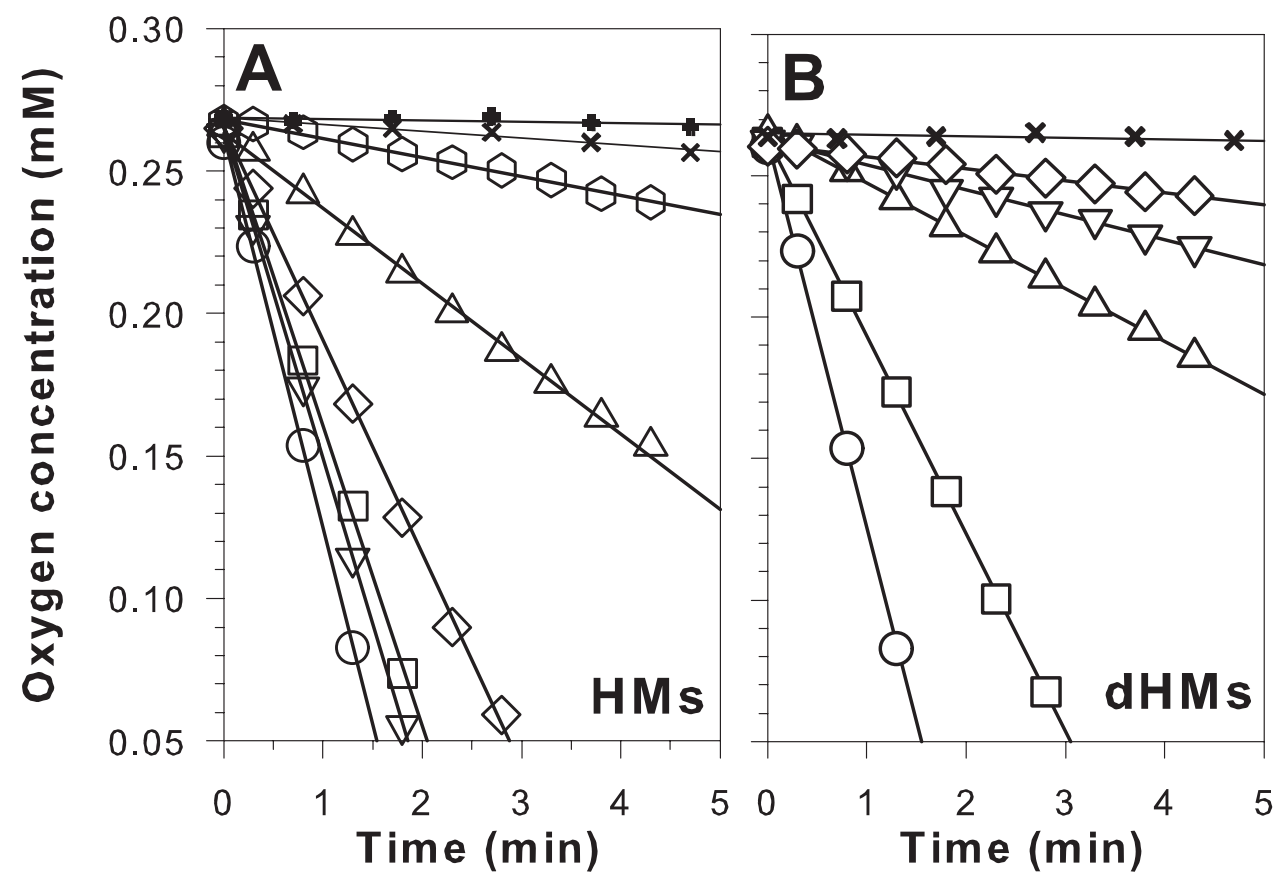

Figure 6. Effects of native (HMs; $\mathbf{A}$, C) and photodegraded for 70 hours (dHMs; B, C) human melanosomes on photosensitized histidine oxidation by TAP. (A, B) Representative kinetics of oxygen depletion in samples containing $0.088 \mathrm{mM}$ TAP and $2 \mathrm{mM}$ histidine, in the presence and absence of human melanosomes at the indicated concentrations (in billion granules/ $\mathrm{mL})$. (C) Mean $( \pm \mathrm{SD})$ initial rates of oxygen uptake as a function of melanosome concentration.

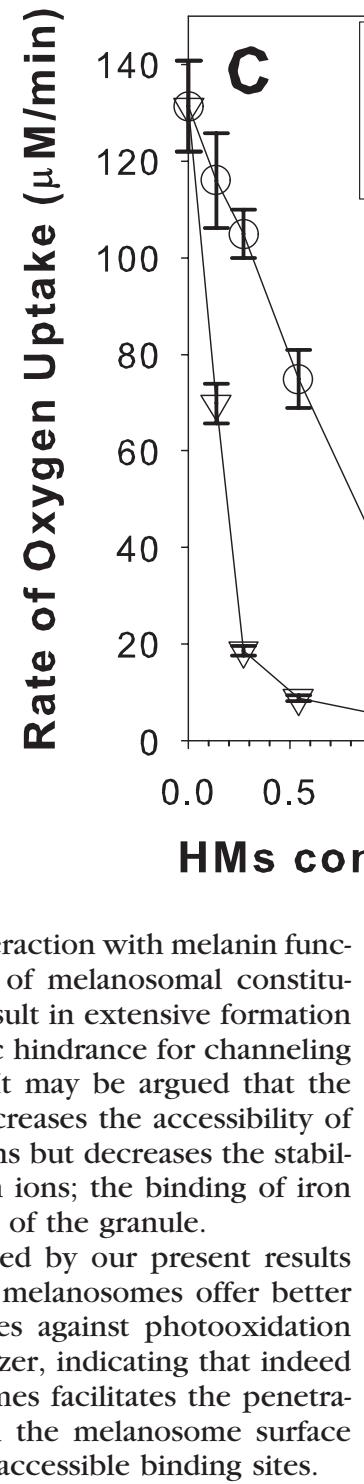

Legend for $A$ and $B$ :

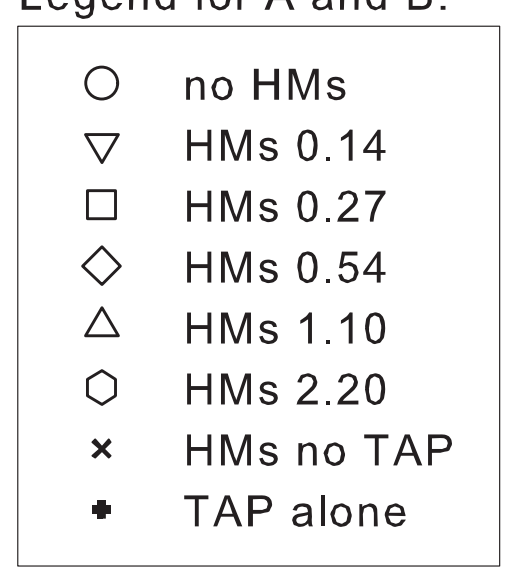

melanin and may modulate their interaction with melanin functional groups. However, oxidation of melanosomal constituents within the granule may also result in extensive formation of cross-links, which impose a steric hindrance for channeling iron ions inside the melanosome. It may be argued that the photooxidation of melanosomes increases the accessibility of some melanin-binding sites to cations but decreases the stability of melanin complexes with iron ions; the binding of iron ions occurs mainly near the surface of the granule.

This scenario is further supported by our present results demonstrating that photodegraded melanosomes offer better protection than native melanosomes against photooxidation mediated by a cationic photosensitizer, indicating that indeed the photodegradation of melanosomes facilitates the penetration of charged molecules through the melanosome surface and increases the concentration of accessible binding sites.
It appears that iron ions bound to the surface of partially degraded melanin are more susceptible to reduction by ascorbate, and their redox cycling elicits oxidation. Such a scenario in which iron binds to degraded melanin but can be easily reduced by ascorbate and the subsequently formed free radicals are scavenged within melanosomes, contributing to oxygen depletion, explains the observed effects by which photodegraded melanosomes appear to be more susceptible than native melanosomes to metal ion-catalyzed oxidation mediated by ascorbate.

\section{Photodegraded Melanosomes Exhibit Increased Antioxidant Protection from Cationic Photosensitizers}

Previously, it had been shown that the cationic photosensitizer TAP binds to synthetic melanin and bovine RPE melano- 
somes $^{4,23,24}$ and that, as a result of the binding, melanin efficiently quenches electronic excited states of TAP. ${ }^{16,25,26}$ We have demonstrated that, in a system in which histidine was used as a singlet oxygen acceptor, the rate of photo-induced oxidation mediated by TAP decreased in the presence of increasing concentrations of bovine RPE melanosomes. ${ }^{4}$ Interestingly, our experiment investigating the effects of human melanosome photodegradation on photosensitized oxidation by a cationic photosensitizer demonstrated that even extensively degraded melanosomes exhibited an increased ability to inhibit oxidation in comparison with native melanosomes. This suggests that the accessibility of the binding sites for the photosensitizer is facilitated by melanosome degradation and that degraded melanin retains its ability to quench excited states of the photosensitizer or singlet oxygen. This may be relevant not only for photosensitizers of extragranular origin but also for lipofuscinlike photosensitizers in melanolipofuscin or melanosomes from aged donors. For instance, a pyridinium bisretinoid (so-called A2E), because of its positive charge, can be expected to bind to melanin. Although A2E exhibits small photoreactivity in comparison with other nonidentified components of lipofuscin, ${ }^{52}$ its binding to melanin may prevent its photodegradation, which is accompanied by the formation of toxic epoxides. ${ }^{53}$ The proximity of melanin to photosensitizers in melanolipofuscin granules may prove protective against photosensitizing action even on extensive degradation of the melanin polymer.

\section{Conclusions}

RPE cells are a site of continual trafficking of iron ions between photoreceptors and the choroidal blood supply. ${ }^{38,39,54}$ Therefore, the ability of RPE melanosomes to sequester iron ions that escape from their binding proteins may have an important physiological role in preventing iron from damaging cellular components of the retinal pigment epithelium. This ability is retained even in aged human melanosomes. However, photodegradation of melanosomes with a physiologically relevant dose of light results in the loss of these antioxidant properties while preserving their ability to bind and deactivate cationic photosensitizers.

Melanin has a large but finite capacity for binding metal ions. ${ }^{32}$ The lifelong accumulation of metal ions within melanosomes may be expected to eventually exceed that capacity, and the protection against iron-mediated oxidation of the retinal pigment epithelium will be diminished. ${ }^{1}$ Moreover, degradation of melanin polymer may be responsible for the loss of protective properties against redox-active iron, and it increases the risk for oxidation of intragranular and extragranular components. An age-related increase in the photogeneration of superoxide radicals ${ }^{10}$ by melanosomes may impose an additional risk for redox cycling of iron ions mediated by the melanosome itself.

Several retinal degenerations have been associated with impairment in iron metabolism and accumulation of excessive amounts of iron in the retina,,$^{38,39,54}$ and iron overload increases susceptibility of the retina to light-induced damage (Qian Y, et al. IOVS 2005; 46:ARVO E-Abstract 1647). Importantly, retinal pigment epithelium affected by age-related macular degeneration (AMD) exhibits approximately fivefold higher concentrations of total iron than age-matched normal retinal pigment epithelium. ${ }^{55}$ In normal and AMD-affected retinal pigment epithelium, approximately half the iron is easily chelatable. ${ }^{55}$ Thus, it is tempting to suggest that age-related changes in melanosomes may be partly responsible for the dysfunction of RPE cells and may be involved in the development of AMD, the primary cause of blindness in the elderly in developed countries.

\section{Acknowledgments}

The authors thank Howard J. Halpern (Chicago University, Chicago, IL) for the MHCTPO.

\section{References}

1. Sarna T. Properties and function of the ocular melanin-a photobiophysical view. J Photochem Photobiol B. 1992;12:215-258.

2. Boulton M. Melanin and the retinal pigment epithelium In: Marmor MF, Wolfensberger TJ, eds. The Retinal Pigment Epitbelium Function and Disease. New York: Oxford University Press; 1998:6885 .

3. Sarna T, Swartz HM. The physical properties of melanins In: Nordlund JJ, Boissy RE, Hearing VJ, King RA, Orthonne J-P, eds. The Pigmentary System Physiology and Patbophysiology. Malden, UK: Blackwell Publishing; 2006:305-335.

4. Sarna T, Rozanowska M, Zareba M, Korytowski W, Boulton M. Retinal melanin and lipofuscin: possible role in photoprotection and phototoxicity of the human RPE. Vienna: 12th International Congress on Pbotobiology-ICP'96; 1998:418-421.

5. Zareba M, Szewczyk G, Sarna T, et al. Effects of photodegradation on the physical and antioxidant properties of melanosomes isolated from retinal pigment epithelium. Pbotochem Pbotobiol. 2006;82:1024-1029.

6. Zadlo A, Rozanowska MB, Burke JM, Sarna TJ. Photobleaching of retinal pigment epithelium melanosomes reduces their ability to inhibit iron-induced peroxidation of lipids. Pigment Cell Res. 2007;20:52-60.

7. Kayatz P, Thumann G, Luther TT, et al. Oxidation causes melanin fluorescence. Invest Ophthalmol Vis Sci. 2001;42:241-246.

8. Sarna T, Burke JM, Korytowski W, et al. Loss of melanin from human RPE with aging: possible role of melanin photooxidation. Exp Eye Res. 2003;76:89-98.

9. Boulton M. Aging of the retinal pigment epithelium. Prog Retinal Res. 1991;11:125-151.

10. Rozanowska M, Korytowski W, Rozanowski B, et al. Photoreactivity of aged human RPE melanosomes: a comparison with lipofuscin. Invest Ophthalmol Vis Sci. 2002;43:2088-2096.

11. Rozanowski B, Cuenco J, Davies S, et al. The phototoxicity of aged human retinal melanosomes. Photochem Pbotobiol. 2008;84:650 657.

12. Sarna T, Pilas B, Land EJ, Truscott TG. Interaction of radicals from water radiolysis with melanin. Biochim Biopbys Acta. 1986;883: 162-167.

13. Lowry OH, Rosebrough NJ, Farr AL, Randall RJ. Protein measurement with the folin phenol reagent. J Biol Chem. 1951;193:265275.

14. Rozanowska M, Jarvis-Evans J, Korytowski W, Boulton ME, Burke JM, Sarna T. Blue light-induced reactivity of retinal age pigment: in vitro generation of oxygen-reactive species. J Biol Cbem. 1995; 270:18825-18830

15. Halpern HJ, Peric M, Nguyen T-D, et al. Selective isotopic labelling of a nitroxide spin label to enhance sensitivity for T2 oximetry. $J$ Magn Reson. 1990;90:40-51.

16. Bielec J, Pilas B, Sarna T, Truscott TG. Photochemical studies of porphyrin-melanin interactions. J Chem Soc Faraday Trans II. 1986;82:1469-1474

17. Davies MJ. Reactive species formed on proteins exposed to singlet oxygen. Photochem Pbotobiol Sci. 2004;3:17-25.

18. Halliwell B, Gutteridge JMC. Free Radicals in Biology and Medicine. 3rd ed. New York: Oxford University Press; 1998.

19. Feeney L. Lipofuscin and melanin of human retinal pigment epithelium: fluorescence, enzyme cytochemical, and ultrastructural studies. Invest Ophthalmol Vis Sci. 1978;17:583-600.

20. Feeney-Burns L, Hilderbrand ES, Eldridge S. Aging human RPE: morphometric analysis of macular, equatorial, and peripheral cells. Invest Opbthalmol Vis Sci. 1984;25:195-200.

21. Weiter JJ, Delori FC, Wing GL, Fitch KA. Retinal pigment epithelial lipofuscin and melanin and choroidal melanin in human eyes. Invest Ophthalmol Vis Sci. 1986;27:145-152.

22. Feeney-Burns L, Burns RP, Gao CL. Age-related macular changes in humans over 90 years old. Am J Opbthalmol. 1990;109:265-278. 
23. Wrobel D, Planner A, Hanyz I, Wielgus A, Sarna T. Melanin-porphyrin interaction monitored by delayed luminescence and photoacoustics. J Photochem Pbotobiol B-Biol. 1997;41:45-52.

24. Wrobel D, Hanyz I, Planner A, Dudkowiak A, Sarna T. Energy deactivation pathways of porphyrins and dopa melanin in polyvinyl alcohol systems. J Photochem Pbotobiol B-Biol. 1998;47:165172 .

25. Bielec J, Pilas B, Sarna T, Knox C, Truscott TG. Photosensitization of melanins covalently bound to dyes. J Chem Soc Faraday Trans II. 1988;84:149-154.

26. Ye T, Simon JD, Sarna T. Ultrafast energy transfer from bound tetra(4-N,N,N,N-trimethylanilinium)porphyrin to synthetic dopa and cysteinyldopa melanins. Photochem Pbotobiol. 2003;77:1-4.

27. Korytowski W, Sarna T, Zareba M. Antioxidant action of neuromelanin-the mechanism of inhibitory effect on lipid peroxidation. Arch Biochem Biophys. 1995;319:142-148.

28. Zareba M, Bober A, Korytowski W, Zecca L, Sarna T. The effect of a synthetic neuromelanin on yield of free hydroxyl radicals generated in model systems. Biochim Biophys Acta-Mol Basis Dis. 1995;1271:343-348.

29. Hong L, Liu Y, Simon JD. Binding of metal ions to melanin and their effects on the aerobic reactivity. Photochem Pbotobiol. 2004;80: 477-481.

30. Rozanowska M, Sarna T, Land EJ, Truscott TG. Free radical scavenging properties of melanin interaction of eu- and pheo-melanin models with reducing and oxidising radicals. Free Radic Biol Med. 1999;26:518-525.

31. Samokhvalov A, Liu Y, Simon JD. Characterization of the Fe(III)binding site in Sepia eumelanin by resonance Raman confocal microspectroscopy. Photochem Pbotobiol. 2004;80:84-88.

32. Liu Y, Hong L, Kempf VR, Wakamatsu K, Ito S, Simon JD. Ionexchange and adsorption of Fe(III) by Sepia melanin. Pigment Cell Res. 2004;17:262-269.

33. Hong L, Simon JD. Current understanding of the binding sites, capacity, affinity, and biological significance of metals in melanin. J Phys Chem B. 2007;111:7938-7947.

34. Pilas B, Sarna T, Kalyanaraman B, Swartz HM. The effect of melanin on iron associated decomposition of hydrogen peroxide. Free Radic Biol Med. 1988;4:285-293.

35. Liu Y, Simon JD. Metal-ion interactions and the structural organization of Sepia eumelanin. Pigment Cell Res. 2005;18:42- 48.

36. Nofsinger JB, Liu Y, Simon JD. Aggregation of eumelanin mitigates photogeneration of reactive oxygen species. Free Radic Biol Med. 2002;32:720-730.

37. Boulton M, Docchio F, Dayhaw-Barker P, Ramponi R, Cubeddu R. Age-related changes in the morphology, absorption and fluorescence of melanosomes and lipofuscin granules of the retinal pigment epithelium. Vision Res. 1990;30:1291-1303.

38. He $\mathrm{X}$, Hahn $\mathrm{P}$, Iacovelli $\mathrm{J}$, et al. Iron homeostasis and toxicity in retinal degeneration. Prog Retin Eye Res. 2007;26:649-673.
39. Dunaief JL. Iron induced oxidative damage as a potential factor in age-related macular degeneration: the Cogan Lecture. Invest $O p b$ thalmol Vis Sci. 2006;47:4660-4664.

40. Yefimova MG, Jeanny JC, Guillonneau X, et al. Iron, ferritin, transferrin, and transferrin receptor in the adult rat retina. Invest $O p b$ thalmol Vis Sci. 2000;41:2343-2351.

41. Yefimova MG, Jeanny JC, Keller N, et al. Impaired retinal iron homeostasis associated with defective phagocytosis in Royal College of Surgeons rats. Invest Opbthalmol Vis Sci. 2002;43:537545 .

42. Ulshafer RJ, Allen CB, Rubin ML. Distributions of elements in the human retinal pigment epithelium. Arch Opbthalmol. 1990;108: 113-117.

43. Eibl O, Schultheiss S, Blitgen-Heinecke P, Schraermeyer U. Quantitative chemical analysis of ocular melanosomes in the TEM. Micron. 2006;37:262-276.

44. Hayasaka S. Aging changes in lipofuscin, lysosomes and melanin in the macular area of human retina and choroid. Jpn J Opbthalmol. 1989;33:36- 42 .

45. Schmidt SY, Peisch RD. Melanin concentration in normal human retinal pigment epithelium-regional variation and age-related reduction. Invest Opbthalmol Vis Sci. 1986;27:1063-1067.

46. Korytowski W, Sarna T. Bleaching of melanin pigments-role of copper ions and hydrogen peroxide in autooxidation and photooxidation of synthetic dopa-melanin. J Biol Chem. 1990;265: 12410-12416.

47. Rozanowska M, Bober A, Burke JM, Sarna T. The role of retinal pigment epithelium melanin in photoinduced oxidation of ascorbate. Pbotochem Pbotobiol. 1997;65:472-479.

48. Burke JM, Henry MM, Zareba M, Sarna T. Photobleaching of melanosomes from retinal pigment epithelium, I: effects on protein oxidation. Photochem Photobiol. 2007;83:920-924.

49. Boettner EA, Wolter JR. Transmission of the ocular media. Invest Ophthalmol. 1962;1:776-783.

50. Sliney DH. Eye protective techniques for bright light. Ophthalmology. 1983;90:937-944.

51. Littrell KC, Gallas JM, Zajac GW, Thiyagarajan P. Structural studies of bleached melanin by synchrotron small-angle X-ray scattering. Photochem Photobiol. 2003;77:115-120.

52. Rozanowska M, Sarna T. Light-induced damage to the retina: role of rhodopsin chromophore revisited. Photochem Pbotobiol. 2005; 81:1305-1330.

53. Sparrow JR, Vollmer-Snarr HR, Zhou JL, et al. A2E-epoxides damage DNA in retinal pigment epithelial cells-vitamin $\mathrm{E}$ and other antioxidants inhibit A2E-epoxide formation. J Biol Chem. 2003; 278:18207-18213.

54. Wong RW, Richa DC, Hahn P, Green WR, Dunaief JL. Iron toxicity as a potential factor in AMD. Retina. 2007;27:997-1003.

55. Hahn P, Milam AH, Dunaief JL. Maculas affected by age-related macular degeneration contain increased chelatable iron in the retinal pigment epithelium and Bruch's membrane. Arch Opbthalmol. 2003;121:1099-1105. 